\title{
Critical role of miRNAs in mediating skeletal muscle atrophy (Review)
}

\author{
YONGHUI YU*, WANLI CHU*, JIAKE CHAI, XIAO LI, LINGYING LIU and LI MA \\ Burn and Plastic Surgery Department, The First Affiliated Hospital to People's Liberation Army General Hospital, \\ Beijing 100048, P.R. China
}

Received February 15, 2015; Accepted December 8, 2015

DOI: $10.3892 / \mathrm{mmr} .2015 .4748$

\begin{abstract}
Skeletal muscle atrophy, a conventional clinical feature in patients with cancer, chronic obstructive pulmonary disease, sepsis and severe burns, is defined as a reduction in muscle mass. During atrophy, the protein degradation is abnormally activated and the aberrance between protein synthesis and protein degradation results in muscle atrophy. Previous studies have demonstrated that miRNAs, small non-coding RNA molecules, serve an important role in the regulation of muscle atrophy. Further studies have indicated the implications of the ubiquitin-proteasome and $\mathrm{PI} 3 \mathrm{~K} / \mathrm{Akt} / \mathrm{FoxO}$ signaling pathways and myogenic regulatory factors in miRNA-mediated muscle atrophy. Therefore, in this review, the effects and molecular mechanisms of miRNAs on muscle atrophy are summarized, leading to the suggestion that miRNAs may serve as potential therapeutic targets in muscle atrophy.
\end{abstract}

\section{Contents}

1. Introduction

2. The effect of the ubiquitin-proteasome pathway on miRNA-mediated muscle atrophy

3. miRNAs mediate muscle atrophy via the regulation of myogenesis

4. Implications of miRNA in cell apoptosis-mediated muscle atrophy

5. PI3K/Akt/FoxO signaling pathway in miRNA-mediated muscle atrophy

6. Conclusion

Correspondence to: Professor Jiake Chai, Burn and Plastic Surgery Department, The First Affiliated Hospital to People's Liberation Army General Hospital, 51 Fucheng Road, Beijing 100048, P.R. China

E-mail: cjk304@126.com

*Contributed equally

Key words: miRNA, protein degradation, differentiation, myogenesis, muscle atrophy

\section{Introduction}

Skeletal muscle makes up approximately $40 \%$ of the body weight and is essential for locomotion (1). Skeletal muscle atrophy, predominantly resulting from excessive protein degradation, occurs in various conditions including starvation (2), aging (3), sepsis (4), cancer cachexia (5), severe burns $(6,7)$ and chronic kidney disease (8). Muscle atrophy results in reductions in mobility of the patients and an increased risk of mortality (9). In patients with severe burns, skeletal muscle atrophy occurs as a result of prolongation of time spent bed-bound and suppression of wound healing (7). In general, skeletal muscle atrophy predicts poor prognosis of patients.

The ubiquitin-proteasome pathway and cell apoptosis are involved in regulating skeletal muscle atrophy (10-12). The ubiquitin-proteasome pathway contributes to protein degradation, as the targeted proteins for degradation are substrates that can be identified and bound to ubiquitin. Subsequently, poly-ubiquitinated substrates are targeted for degradation by proteasomes (11). Activation of the ubiquitin-proteasome pathway may serve an important role in the mediation of skeletal muscle atrophy $(10,11)$. Previous studies have additionally demonstrated that increased cell apoptosis is accompanied by stress-induced skeletal muscle atrophy (12), and that apoptosis is also a critical factor which leads to muscle atrophy $(13,14)$.

MicroRNAs (miRNAs), the small non-coding RNAs, were first identified in $C$. elegans and are highly conserved in eukaryotes (15). At present, greater than 1,700 miRNAs have been identified, which serve critical roles in regulating proliferation, differentiation and the development of various diseases (16). miRNA exerts its biological activation via binding to the 3'-untranslated region (3'-UTR) of targeted mRNA (17). Accelerating target mRNA degradation or inhibiting its translation are two key ways in which miRNA mediates the control of gene expression (18). The miRNA (miR)-1/206 family, miR-133, miR-208 and miR-488 are identified as muscle-specific miRNAs and serve essential roles in regulating normal myoblast differentiation, proliferation and muscle remodeling in response to stress (19-21). In addition, muscle-specific miRNAs, miR-128a and miR-351, are involved in the regulation of myogenesis $(21,22)$.

The present review will focus upon the miRNAs involved in the regulation of skeletal muscle atrophy and the potential molecular mechanisms. Further studies are required in order 
to elucidate the specific miRNAs implicated in stress-induced skeletal muscle atrophy, which may lead to the development of novel targets for clinical therapy.

\section{The effect of the ubiquitin-proteasome pathway on miR- NA-mediated muscle atrophy}

Aberrant activation of protein degradation is the key factor that leads to muscle atrophy, and the ubiquitin-proteasome pathway serves a pivotal role in the mediation of protein degradation (11). The proteasome can identify the poly-ubiquitinated protein and trigger the degradation procedure (23). E3 ligase is the critical mediator of protein ubiquitination. Muscle RING finger 1 (MuRF1) and muscle atrophy F-box (MAFbx) are two muscle specific E3 ligases (24). During muscle atrophy, MuRF1 and MAFbx are overexpressed in muscle, and inhibiting the function of MuRF1 and MAFbx has been demonstrated to suppress muscle loss and subsequently attenuate muscle atrophy $(25,26)$. Previous studies (27-29) have additionally indicated that miRNAs are implicated in the regulation of MuRF1 and MAFbx expression (Fig. 1). miR-23a is able to inhibit the translational activation of MuRF1 and MAFbx via binding with their 3'-UTR, and miR-23a transgenic mice exert resistance against glucocorticoid-induced muscle atrophy (27). In a dexamethasone (Dex)-induced mouse model of atrophy, muscle-specific miR-1 expression is upregulated. miR-1 has been previously reported to induce MuRF1 and MAFbx expression via the HSP70/protein kinase B(Akt)/forkhead box (Fox) O3 signaling pathway and is responsible for Dex-induced muscle atrophy (28). The miR-199/214 cluster is also involved in regulating the ubiquitin-proteasome pathway (29). Taken together, miRNA-dependent activation of the ubiquitin-proteasome pathway is responsible for the promotion of muscle atrophy by directly or indirectly targeting the muscle specific E3 ligases of MuRF1 and MAFbx.

\section{3. miRNAs mediate muscle atrophy via the regulation of myogenesis}

In addition to enhancing muscle proteolysis, aberrant myogenesis is also a critical factor during muscle atrophy. Myogenesis is impaired in models of mice with cancer (30), and pigs with chronic obstructive pulmonary disease (31). Inactivation of myogenesis is also observed in diseases such as Duchenne muscular dystrophy and spinal and bulbar muscular atrophy $(32,33)$. Muscle satellite cells are stem cells with self-renewal and differentiation potency, and when muscle disruption occurs, proliferative satellite cells could differentiate into myotubes and contribute to muscle regeneration (34). Defects in post-natal myogenesis and muscle regeneration result in muscle atrophy, and miRNAs are implicated in the regulation of myogenesis and muscle atrophy $(35,36)$. As presented in Fig. 2, paired-box transcription factor (Pax) is essential for satellite cell proliferation and differentiation. miR-1, miR-206 and miR-486 restrict satellite cell proliferation and promote its differentiation through suppression of Pax7 expression (37-39). Pax3 is also the critical factor required to trigger satellite cell proliferation; suppressing miR-27b, miR-1 and miR-206 expression suppresses satellite cell differentiation via enhancement of Pax3 activation $(40,41)$. The myogenic regulatory factor (MRF) family, which includes MyoD, Myf5, myogenin and Myf6, has the pivotal role in myogenic differentiation. MyoD is expressed in activated satellite cells, and miR-27a overexpression elevates the MyoD protein level and enhances myoblast differentiation (42). In C2C12 myoblast cells, miR-26a upregulates MyoD expression and promotes the myogenic process (43). Subsequent to injury, miR-26a is induced during muscle regeneration, and blocking miR-26a expression enhances Smad-dependent muscle differentiation (44). miR-186 suppresses C2C12 myoblast cell myogenic differentiation via targeting myogenin (45). In addition to the MRF family, miRNAs also regulate myogenesis through targeting a variety of proteins. Myostatin is the negative mediator of myogenesis; miR-27a and miR-27b promote satellite cell proliferation and post-natal myogenesis by suppressing myostatin expression (46-48). miR-125b, miR-133 and miR-199a-3p are involved in the regulation of the insulin-like growth factor/insulin-like growth factor receptor signaling pathway and inhibit cell differentiation and muscle regeneration (49-51). miR-203 functions as the suppressor of myoblast differentiation by repressing c-Jun and myocyte enhancer factor 2C (MEF2C) expression (52). miR-155 inhibits MEF2A expression and suppresses the myogenic process (53). miR-29 is a pro-myogenic factor, which acts through downregulation of Akt3 or RING1 and YY1-binding protein $(54,55)$. Thus, miRNAs have critical roles in regulating satellite cell proliferation, myogenic differentiation and muscle regeneration.

\section{Implications of miRNAs in cell apoptosis-mediated mus- cle atrophy}

Cell apoptosis is programmed cell death and is a promoting factor of muscle atrophy $(14,56)$. Studies using a mouse model have demonstrated that cell apoptosis is involved in the progression of heart failure, severe burns and age-associated muscle atrophy (57-60). The mitochondria and caspase-mediated apoptotic pathways are some of the mechanisms of burn, age or stress-induced muscle atrophy $(12,57,61,62)$. miRNA is an important mediator of myoblast cell apoptosis (63). In skeletal muscle, pre-conditional activation of interleukin (IL)-11/signal transducer and activator of transcription (STAT)3 pathway protects human skeletal myoblasts from oxidant-induced apoptosis (64), and miR-21 is a key regulator of extracellular signal-related kinase 1/2-STAT3 signaling downstream of IL-11 and inhibits the apoptosis of skeletal myoblasts (65). Skeletal muscle loss in cancer cachexia is partially associated with cell apoptosis, and a previous study indicated that miR-21 in microvesicles of cancer cachexia triggers muscle cell apoptosis via enhancement of c-Jun N-terminal kinase activation (66). In acute muscle injury, myogenic progenitor cell apoptosis is triggered by miR-351 knockdown (21). MyoD is a critical factor in the regulation muscle differentiation; MyoD knockout in myoblasts decelerates miR-1 and miR-206 expression and results in resistance to apoptosis (67). Forced MyoD expression in MyoD knockout myoblasts enhances the expression of miR-1 and miR-206 and triggers cell apoptosis via Pax3 downregulation (67). Thus, it is suggested that miRNA is a critical mediator in regulating myoblast apoptosis and implicated in muscle atrophic process. 


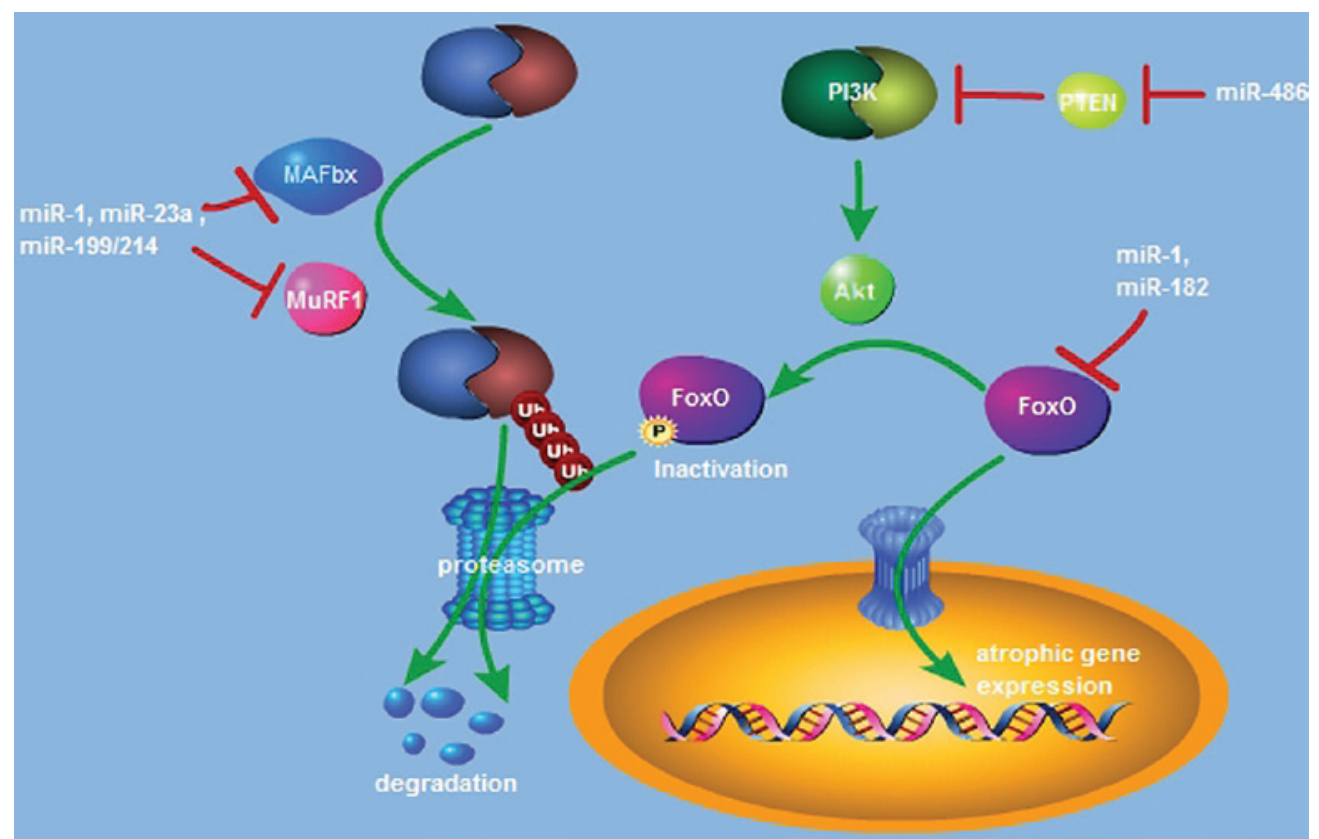

Figure 1. Schematic of the involvement of miRs in regulating muscle protein degradation and atrophic gene expression. MuRF1 and MAFbx are critical E3 ligases for mediating muscle protein ubiquitin, and ubiquitinated protein is degraded by proteasomes. miR-1, miR-182 and the miR-192/214 cluster exert inhibition of protein degradation by targeting MuRF1 and MAFbx. miRNAs are also implicated in the regulation of atrophic gene expression in a $\mathrm{PI} 3 \mathrm{~K} / \mathrm{Akt} /$ FoxO-dependent manner. PTEN, the phosphatase of PI3K, is the target of miR-486. Suppressing PTEN expression enhances PI3K/Akt activation and promotes FoxO phosphorylation. Phosphorylated FoxO is located in the cytoplasm and is degraded by the proteasome. miR-1 and miR-182 are able to repress atrophic gene expression via inhibition of FoxO protein translation. miR, microRNA; MuRF1, muscle RING finger 1; MAFbx, muscle atrophy F-box; PI3K, phosphoinositide 3-kinase; Akt, protein kinase B; FoxO, forkhead box O; PTEN, phosphatase and tensin homolog.

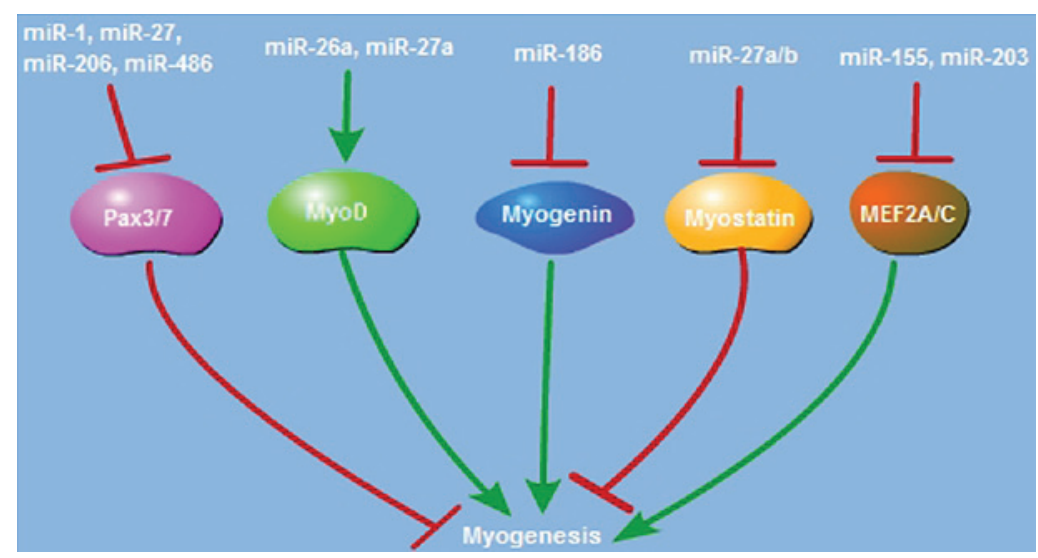

Figure 2. miRs and myogenesis regulation. The transcription factors Pax3/7 are essential for the maintenance of satellite cell proliferation and suppression of the myogenic process. Myostatin also functions as the suppressor of myogenesis. miR-1, miR-27, miR-206 and miR-486 are implicated in restriction of Pax3/7 or myostatin expression, respectively. Myogenic regulator factors, such as $\mathrm{MyoD}$ and myogenin, trigger myogenic process and are regulated by miR-26a, miR-27a and miR-186. miR-155 and miR-203 also suppress myogenesis by targeting MEF2A/C. miR, microRNA; Pax3/7, paired-box 3/7; MEF2A/C, myocyte enhancer factor $2 \mathrm{~A}$.

\section{PI3K/Akt/FoxO signaling pathway in miRNA-mediated muscle atrophy}

The PI3K/Akt/FoxO signal pathway serves an important role in muscle atrophy (Fig. 1). Attenuated activation of the PI3K/Akt signaling pathway results in rat skeletal muscle atrophy $(13,68)$. Akt inactivation functions as the promoter of burn-induced muscle atrophy (69). FoxO is phosphorylated and exported into the cytoplasm by the upstream kinase Akt, and cytoplasmic FoxO is degraded with loss of transactivation (70). FoxO is additionally implicated in promoting muscle atrophy (71). Tumor necrosis factor receptor-associated factor 6 promotes starvation-induced atrophy in an Akt/FoxO3a-dependent manner (2). FoxO1, the dominant mediator of muscle atrophy, serves a critical role in chronic kidney disease or burn-induced muscle atrophy $(8,72)$. miR-486, the regulator of PTEN, is overexpressed in Duchenne muscular dystrophy; and miR-486 transgenic mice exert the impairment of muscle regeneration in a PTEN/Akt dependent manner (73). In patients with breast patients, miR-486 is downregulated, and the expression of its target genes PTEN and FoxO1A are elevated (74). Myostatin is well known as a negative regulator of muscle mass by reducing protein synthesis. Overexpression of miR-486 is observed in skeletal muscle of myostatin knockout mice and is essential to maintain skeletal 
muscle size through the Akt/mTOR signaling pathway (75). In the $\mathrm{C} 2 \mathrm{C} 12$ myotube cells, $\mathrm{miR}-182$ was reported to suppress FoxO3a protein expression via binding to the 3'-UTR of FoxO3a mRNA, and prevent glucocorticoid-induced rat muscle atrophy (76). Muscle-specific miR-1 is involved in dephosphorylating and activating FoxO3a in an HSP70/Akt dependent manner and promotes Dex- or myostatin-induced atrophy in skeletal muscle (28). In summary, PI3K/Akt inactivation reduces FoxO protein phosphorylation and dephosphorylated FoxO enters into the nucleus and promotes muscle atrophy.

\section{Conclusion}

Aberrant muscle protein degradation, impairment of myogenesis, and promotion of muscle cell apoptosis are all important factors that contribute to muscle atrophy. miRNAs are critical mediators of protein degradation and myogenesis through regulation of the ubiquitin-proteasome and PI3K/Akt/FoxO signaling pathways and other myogenic regulatory factors. Thus, miRNAs may be potential and effective therapeutic targets for muscle atrophy.

\section{Acknowledgements}

The authors would like to thank Dr Hongli Wu for her critical reading of this review. The current study was supported by the National Science Foundation of China (grant nos. NSFC81120108014, NSFC81471873, NSFC81501694, NSFC81571894 and NSFC81171807), the Beijing Natural Science Foundation (grant no. 7144250) and the Nursery Fund of People's Liberation Army General Hospital (grant nos. 14KMM22 and 13KMM31).

\section{References}

1. Hitachi K and Tsuchida K: Role of microRNAs in skeletal muscle hypertrophy. Front Physiol 4: 408, 2014.

2. Paul PK, Bhatnagar S, Mishra V, Srivastava S, Darnay BG, Choi Y and Kumar A: The E3 Kubiquitin ligase TRAF6 intercedes in starvation-induced skeletal muscle atrophy through multiple mechanisms. Mol Cell Biol 32: 1248-1259, 2012.

3. McGregor RA, Poppitt SD and Cameron-Smith D: Role of microRNAs in the age-related changes in skeletal muscle and diet or exercise interventions to promote healthy aging in humans. Ageing Res Rev 17: 25-33, 2014.

4. Nystrom G, Pruznak A, Huber D, Frost RA and Lang CH: Local insulin-like growth factor I prevents sepsis-induced muscle atrophy. Metabolism 58: 787-797, 2009.

5. Wang H, Lai YJ, Chan YL, Li TL and Wu CJ: Epigallocatechin-3-gallate effectively attenuates skeletal muscle atrophy caused by cancer cachexia. Cancer Lett 305: 40-49, 2011.

6. Bertsch S, Lang $\mathrm{CH}$ and Vary TC: Inhibition of glycogen synthase kinase 3[beta] activity with lithium in vitro attenuates sepsis-induced changes in muscle protein turnover. Shock 35 : 266-274, 2011.

7. Hart DW, Wolf SE, Chinkes DL, Gore DC, Mlcak RP, Beauford RB, Obeng MK, Lal S, Gold WF, Wolfe RR and Herndon DN: Determinants of skeletal muscle catabolism after severe burn. Ann Surg 232: 455-465, 2000.

8. Xu J, Li R, Workeneh B, Dong Y, Wang X and Hu Z: Transcription factor FoxO1, the dominant mediator of muscle wasting in chronic kidney disease, is inhibited by microRNA-486. Kidney Int 82: 401-411, 2012.

9. Metter EJ, Talbot LA, Schrager M and Conwit R: Skeletal muscle strength as a predictor of all-cause mortality in healthy men J Gerontol A Biol Sci Med Sci 57: B359-B365, 2002.

10. Chai J, Wu Y and Sheng ZZ: Role of ubiquitin-proteasome pathway in skeletal muscle wasting in rats with endotoxemia. Crit Care Med 31: 1802-1807, 2003.
11. Attaix D, Combaret L, Bechet D and Taillandier D: Role of the ubiquitin-proteasome pathway in muscle atrophy in cachexia. Curr Opin Support Palliat Care 2: 262-266, 2008.

12. Sishi B,Loos B,Ellis B, Smith W, du Toit EF and Engelbrecht AM: Diet-induced obesity alters signalling pathways and induces atrophy and apoptosis in skeletal muscle in a prediabetic rat model. Exp Physiol 96: 179-193, 2011.

13. Engelbrecht AM, Smith C, Neethling I, Thomas M, Ellis B, Mattheyse $\mathrm{M}$ and Myburgh KH: Daily brief restraint stress alters signaling pathways and induces atrophy and apoptosis in rat skeletal muscle. Stress 13: 132-141, 2010.

14. Dupont-Versteegden EE: Apoptosis in skeletal muscle and its relevance to atrophy. World J Gastroenterol 12: 7463-7466, 2006.

15. Lee RC, Feinbaum RL and Ambros V: The C. elegans heterochronic gene lin-4 encodes small RNAs with antisense complementarity to lin-14. Cell 75: 843-854, 1993.

16. Sayed D and Abdellatif M: MicroRNAs in development and disease. Physiol Rev 91: 827-887, 2011.

17. Didiano D and Hobert O: Molecular architecture of a miRNA-regulated 3' UTR. RNA 14: 1297-1317, 2008.

18. Bartel DP: MicroRNAs: Target recognition and regulatory functions. Cell 136: 215-233, 2009.

19. Travaglini L, Vian L, Billi M, Grignani F and Nervi C: Epigenetic reprogramming of breast cancer cells by valproic acid occurs regardless of estrogen receptor status. Int J Biochem Cell Biol 41: 225-234, 2009.

20. Taulli R, Bersani F, Foglizzo V, Linari A, Vigna E, Ladanyi M, Tuschl T and Ponzetto C: The muscle-specific microRNA miR-206 blocks human rhabdomyosarcoma growth in xenotransplanted mice by promoting myogenic differentiation. J Clin Invest 119: 2366-2378, 2009.

21. Chen Y, Melton DW, Gelfond JA, McManus LM and Shireman PK: MiR-351 transiently increases during muscle regeneration and promotes progenitor cell proliferation and survival upon differentiation. Physiol Genomics 44: 1042-1051, 2012.

22. Motohashi N, Alexander MS, Shimizu-Motohashi Y, Myers JA, Kawahara G and Kunkel LM: Regulation of IRS1/Akt insulin signaling by microRNA-128a during myogenesis. J Cell Sci 126: 2678-2691, 2013

23. Hartmann-Petersen R and Gordon C: Proteins interacting with the 26S proteasome. Cell Mol Life Sci 61: 1589-1595, 2004.

24. Bodine SC, Latres E, Baumhueter S, Lai VK, Nunez L, Clarke BA, Poueymirou WT, Panaro FJ, Na E, Dharmarajan K, et al: Identification of ubiquitin ligases required for skeletal muscle atrophy. Science 294: 1704-1708, 2001.

25. Eddins MJ, Marblestone JG, Suresh Kumar KG, Leach CA, Sterner DE, Mattern MR and Nicholson B: Targeting the ubiquitin E3 ligase MuRF1 to inhibit muscle atrophy. Cell Biochem Biophys 60: 113-118, 2011.

26. Clavel S, Coldefy AS, Kurkdjian E, Salles J, Margaritis I and Derijard B: Atrophy-related ubiquitin ligases, atrogin-1 and MuRF1 are up-regulated in aged rat Tibialis Anterior muscle. Mech Ageing Dev 127: 794-801, 2006.

27. Wada S, Kato Y, Okutsu M, Miyaki S, Suzuki K, Yan Z, Schiaffino S, Asahara H, Ushida T and Akimoto T: Translational suppression of atrophic regulators by microRNA-23a integrates resistance to skeletal muscle atrophy. J Biol Chem 286: 38456-38465, 2011.

28. Kukreti H, Amuthavalli K, Harikumar A, Sathiyamoorthy S, Feng PZ, Anantharaj R, Tan SL, Lokireddy S, Bonala S, Sriram S, et al: Muscle-specific microRNA1 (miR1) targets heat shock protein 70 (HSP70) during dexamethasone-mediated atrophy. J Biol Chem 288: 6663-6678, 2013.

29. Baumgarten A, Bang C, Tschirner A, Engelmann A, Adams V, von Haehling S, Doehner W, Pregla R, Anker MS, Blecharz K, et al: TWIST1 regulates the activity of ubiquitin proteasome system via the miR-199/214 cluster in human end-stage dilated cardiomyopathy. Int J Cardiol 168: 1447-1452, 2013.

30. Penna F, Costamagna D, Fanzani A, Bonelli G, Baccino FM and Costelli P: Muscle wasting and impaired myogenesis in tumor bearing mice are prevented by ERK inhibition. PLoS One 5: e13604, 2010

31. Verhees KJ, Pansters NA, Baarsma HA, Remels AH, Haegens A, de Theije CC, Schols AM, Gosens R and Langen RC: Pharmacological inhibition of GSK-3 in a guinea pig model of LPS-induced pulmonary inflammation: II. Effects on skeletal muscle atrophy. Respir Res 14: 117, 2013.

32. Shi H, Verma M, Zhang L, Dong C, Flavell RA and Bennett AM: Improved regenerative myogenesis and muscular dystrophy in mice lacking Mkp5. J Clin Invest 123: 2064-2077, 2013. 
33. Malena A, Pennuto M, Tezze C, Querin G, D'Ascenzo C, Silani V, Cenacchi G, Scaramozza A, Romito S, Morandi L, et al: Androgen-dependent impairment of myogenesis in spinal and bulbar muscular atrophy. Acta Neuropathol 126: 109-121, 2013.

34. Sacco A, Doyonnas R, Kraft P, Vitorovic S and Blau HM: Self-renewal and expansion of single transplanted muscle stem cells. Nature 456: 502-506, 2008.

35. Dachs E, Hereu M, Piedrafita L, Casanovas A, Calderó J and Esquerda JE: Defective neuromuscular junction organization and postnatal myogenesis in mice with severe spinal muscular atrophy. J Neuropathol Exp Neurol 70: 444-461, 2011

36. Wang XH: MicroRNA in myogenesis and muscle atrophy. Curr Opin Clin Nutr Metab Care 16: 258-266, 2013

37. Chen JF, Tao Y, Li J, Deng Z, Yan Z, Xiao X and Wang DZ: microRNA-1 and microRNA-206 regulate skeletal muscle satellite cell proliferation and differentiation by repressing Pax7. J Cell Biol 190: 867-879, 2010.

38. Dey BK, Gagan J and Dutta A: miR-206 and -486 induce myoblast differentiation by downregulating Pax7. Mol Cell Biol 31: 203-214, 2011.

39. Liu N, Williams AH, Maxeiner JM, Bezprozvannaya S, Shelton JM, Richardson JA, Bassel-Duby R and Olson EN microRNA-206 promotes skeletal muscle regeneration and delays progression of Duchenne muscular dystrophy in mice. J Clin Invest 122: 2054-2065, 2012.

40. Goljanek-Whysall K, Sweetman D, Abu-Elmagd M, Chapnik E, Dalmay T, Hornstein E and Münsterberg A: MicroRNA regulation of the paired-box transcription factor Pax3 confers robustness to developmental timing of myogenesis. Proc Natl Acad Sci USA 108: 11936-11941, 2011.

41. Crist CG, Montarras D, Pallafacchina G, Cumano A, Conway SJ and Buckingham M: Muscle stem cell behavior is modified by microRNA-27 regulation of Pax3 expression. Proc Natl Acad Sci USA 106: 13383-13387, 2009.

42. Chen X, Huang Z, Chen D, Yang T and Liu G: Role of microRNA-27a in myoblast differentiation. Cell Biol Int 38: 266-271, 2014.

43. Wong CF and Tellam RL: MicroRNA-26a targets the histone methyltransferase Enhancer of Zeste homolog 2 during myogenesis. J Biol Chem 283: 9836-9843, 2008.

44. Dey BK, Gagan J, Yan Z and Dutta A: miR-26a is required for skeletal muscle differentiation and regeneration in mice. Genes Dev 26: 2180-2191, 2012.

45. Antoniou A, Mastroyiannopoulos NP, Uney JB and Phylactou LA: miR-186 inhibits muscle cell differentiation through myogenin regulation. J Biol Chem 289: 3923-3935, 2014.

46. Huang Z, Chen X, Yu B, He J and Chen D: MicroRNA-27a promotes myoblast proliferation by targeting myostatin. Biochem Biophys Res Commun 423: 265-269, 2012.

47. McFarlane C, Vajjala A, Arigela H, Lokireddy S, Ge X, Bonala S, Manickam R, Kambadur R and Sharma M: Negative auto-regulation of myostatin expression is mediated by $\mathrm{Smad} 3$ and microRNA-27. PLoS One 9: e87687, 2014.

48. Yang T, Chen XL, Huang ZQ, Wen WX, Xu M, Chen DW, Yu B, He J, Luo JQ, Yu J, et al: MicroRNA-27a promotes porcine myoblast proliferation by downregulating myostatin expression. Animal 8: 1867-1872, 2014.

49. Ge Y, Sun Y and Chen J: IGF-II is regulated by microRNA-125b in skeletal myogenesis. J Cell Biol 192: 69-81, 2011.

50. Huang MB, Xu H, Xie SJ, Zhou $\mathrm{H}$ and $\mathrm{Qu} \mathrm{LH}$ : Insulin-like growth factor-1 receptor is regulated by microRNA-133 during skeletal myogenesis. PLoS One 6: e29173, 2011.

51. Jia L, Li YF, Wu GF, Song ZY, Lu HZ, Song CC, Zhang QL, Zhu JY, Yang GS and Shi XE: MiRNA-199a-3p regulates C2C12 myoblast differentiation through IGF-1/AKT/mTOR signal pathway. Int J Mol Sci 15: 296-308, 2013.

52. Luo W, Wu H, Ye Y, Li Z, Hao S, Kong L, Zheng X, Lin S, Nie $\mathrm{Q}$ and Zhang X: The transient expression of miR-203 and its inhibiting effects on skeletal muscle cell proliferation and differentiation. Cell Death Dis 5: e1347, 2014.

53. Seok HY, Tatsuguchi M, Callis TE, He A, Pu WT and Wang DZ: miR-155 inhibits expression of the MEF2A protein to repress skeletal muscle differentiation. J Biol Chem 286: 35339-35346, 2011

54. Wei W, He HB, Zhang WY, Zhang HX, Bai JB, Liu HZ, Cao JH, Chang KC, Li XY and Zhao SH: miR-29 targets Akt3 to reduce proliferation and facilitate differentiation of myoblasts in skeletal muscle development. Cell Death Dis 4: e668, 2013.

55. Zhou L, Wang L, Lu L, Jiang P, Sun H and Wang H: A novel target of microRNA-29, Ring1 and YY1-binding protein (Rybp), negatively regulates skeletal myogenesis. J Biol Chem 287: 25255-25265, 2012.
56. Dupont-Versteegden EE: Apoptosis in muscle atrophy: Relevance to sarcopenia. Exp Gerontol 40: 473-481, 2005.

57. Dirks AJ and Leeuwenburgh C: The role of apoptosis in age-related skeletal muscle atrophy. Sports Med 35: 473-483, 2005.

58. Lee HY, Kaneki M, Andreas J, Tompkins RG and Martyn JA Novel mitochondria-targeted antioxidant peptide ameliorates burn-induced apoptosis and endoplasmic reticulum stress in the skeletal muscle of mice. Shock 36: 580-585, 2011.

59. Fanzani A, Conraads VM, Penna F and Martinet W: Molecular and cellular mechanisms of skeletal muscle atrophy: An update. J Cachexia Sarcopenia Muscle 3: 163-179, 2012.

60. Libera LD, Zennaro R, Sandri M, Ambrosio GB and Vescovo G: Apoptosis and atrophy in rat slow skeletal muscles in chronic heart failure. Am J Physiol 277: C982-C986, 1999.

61. Yasuhara S, Perez ME, Kanakubo E, Yasuhara Y, Shin YS, Kaneki M, Fujita T and Martyn JA: Skeletal muscle apoptosis after burns is associated with activation of proapoptotic signals. Am J Physiol Endocrinol Metab 279: E1114-E1121, 2000.

62. Marzetti E, Lawler JM, Hiona A, Manini T, Seo AY and Leeuwenburgh C: Modulation of age-induced apoptotic signaling and cellular remodeling by exercise and calorie restriction in skeletal muscle. Free Radic Biol Med 44: 160-168, 2008.

63. Callis TE, Chen JF and Wang DZ: MicroRNAs in skeletal and cardiac muscle development. DNA Cell Biol 26: 219-225, 2007.

64. Idris NM, Ashraf M, Ahmed RP, Shujia J and Haider KH: Activation of IL-11/STAT3 pathway in preconditioned human skeletal myoblasts blocks apoptotic cascade under oxidant stress. Regen Med 7: 47-57, 2012.

65. Haider KH, Idris NM, Kim HW, Ahmed RP, Shujia J and Ashraf M: MicroRNA-21 is a key determinant in IL-11/Stat3 anti-apoptotic signalling pathway in preconditioning of skeletal myoblasts. Cardiovasc Res 88: 168-178, 2010.

66. He WA, Calore F, Londhe P, Canella A, Guttridge DC and Croce CM: Microvesicles containing miRNAs promote muscle cell death in cancer cachexia via TLR7. Proc Natl Acad Sci USA 111: 4525-4529, 2014

67. Hirai H, Verma M, Watanabe S, Tastad C, Asakura Y and Asakura A: MyoD regulates apoptosis of myoblasts through microRNA-mediated down-regulation of Pax3. J Cell Biol 191: 347-365, 2010.

68. Stitt TN, Drujan D, Clarke BA, Panaro F, Timofeyva Y, Kline WO, Gonzalez M, Yancopoulos GD and Glass DJ: The IGF-1/PI3K/Akt pathway prevents expression of muscle atrophy-induced ubiquitin ligases by inhibiting FOXO transcription factors. Mol Cell 14: 395-403, 2004.

69. Sugita H, Kaneki M, Sugita M, Yasukawa T, Yasuhara S and Martyn JA: Burn injury impairs insulin-stimulated Akt/PKB activation in skeletal muscle. Am J Physiol Endocrinol Metab 288: E585-E591, 2005.

70. Du K, Yu Y,Zhang D, Luo W, Huang H, Chen J, Gao J and Huang C: NFkappaB1 (p50) suppresses SOD2 expression by inhibiting FoxO3a transactivation in a miR190/PHLPP1/Akt-dependent axis. Mol Biol Cell 24: 3577-3583, 2013.

71. Sandri M, Sandri C, Gilbert A, Skurk C, Calabria E, Picard A, Walsh K, Schiaffino S, Lecker SH and Goldberg AL: Foxo transcription factors induce the atrophy-related ubiquitin ligase atrogin-1 and cause skeletal muscle atrophy. Cell 117: 399-412, 2004.

72. Sheriff S, Kadeer N, Joshi R, Friend LA, James JH and Balasubramaniam A: Des-acyl ghrelin exhibits pro-anabolic and anti-catabolic effects on $\mathrm{C} 2 \mathrm{C} 12$ myotubes exposed to cytokines and reduces burn-induced muscle proteolysis in rats. Mol Cell Endocrinol 351: 286-295, 2012

73. Alexander MS, Casar JC, Motohashi N, Myers JA, Eisenberg I, Gonzalez RT, Estrella EA, Kang PB, Kawahara G and Kunkel LM: Regulation of DMD pathology by an ankyrin-encoded miRNA. Skelet Muscle 1: 27, 2011.

74. Chen D, Goswami CP, Burnett RM, Anjanappa M, Bhat-Nakshatri P, Muller W and Nakshatri H: Cancer affects microRNA expression, release and function in cardiac and skeletal muscle. Cancer Res 74: 4270-4281, 2014.

75. Hitachi K, Nakatani M and Tsuchida K: Myostatin signaling regulates Akt activity via the regulation of miR-486 expression. Int J Biochem Cell Biol 47: 93-103, 2014.

76. Hudson MB, Rahnert JA, Zheng B, Woodworth-Hobbs ME, Franch HA and Price SR: miR-182 attenuates atrophy-related gene expression by targeting FoxO3 in skeletal muscle. Am J Physiol Cell Physiol 307: C314-C319, 2014. 